University of Nebraska - Lincoln DigitalCommons@University of Nebraska - Lincoln

CSE Journal Articles

Computer Science and Engineering, Department of

2014

\title{
A Statistical-Physical Model of Interference in Diffusion-Based Molecular Nanonetworks
}

Massimiliano Pierobon

University of Nebraska-Lincoln, pierobon@cse.unl.edu

Ian F. Akyildiz

Georgia Institute of Technology

Follow this and additional works at: http://digitalcommons.unl.edu/csearticles

Pierobon, Massimiliano and Akyildiz, Ian F., "A Statistical-Physical Model of Interference in Diffusion-Based Molecular

Nanonetworks" (2014). CSE Journal Articles. 121.

http://digitalcommons.unl.edu/csearticles/121

This Article is brought to you for free and open access by the Computer Science and Engineering, Department of at DigitalCommons@University of Nebraska - Lincoln. It has been accepted for inclusion in CSE Journal Articles by an authorized administrator of DigitalCommons@University of

Nebraska - Lincoln. 


\title{
A Statistical-Physical Model of Interference in Diffusion-Based Molecular Nanonetworks
}

\author{
Massimiliano Pierobon, Member, IEEE, and Ian F. Akyildiz, Fellow, IEEE
}

\begin{abstract}
Molecular nanonetworks stand at the intersection of nanotechnology, biotechnology, and network engineering. The research on molecular nanonetworks proposes the interconnection of nanomachines through molecule exchange. Amongst different solutions for the transport of molecules between nanomachines, the most general is based on free diffusion. The objective of this paper is to provide a statistical-physical modeling of the interference when multiple transmitting nanomachines emit molecules simultaneously. This modeling stems from the same assumptions used in interference study for radio communications, namely, a spatial Poisson distribution of transmitters having independent and identically distributed emissions, while the specific molecule emissions model is in agreement with a chemical description of the transmitters. As a result of the property of the received molecular signal of being a stationary Gaussian Process (GP), the statistical-physical modeling is operated on its Power Spectral Density (PSD), for which it is possible to obtain an analytical expression of the log-characteristic function. This expression leads to the estimation of the received PSD probability distribution, which provides a complete model of the interference in diffusion-based molecular nanonetworks. Numerical results in terms of received PSD probability distribution and probability of interference are presented to compare the proposed statistical-physical model with the outcomes of simulations.
\end{abstract}

Index Terms-Co-channel interference, diffusion equation, molecular communication (MC), nanonetworks, statisticalphysical interference model.

\section{INTRODUCTION}

$\mathbf{M}$ OLECULAR nanonetworks are one of the new frontiers in communication engineering and networking. Proposed for the interconnection of intelligent autonomous nanodevices, or nanomachines, they are based on a bio-inspired paradigm called Molecular Communication (MC) [1]. MC realizes information transmission between devices through the exchange of molecules, which are emitted by a transmitting device, propagated through different techniques and received by the destination device. This new paradigm, inspired by the

Manuscript received June 3, 2013; revised November 10, 2013; accepted March 11, 2014. Date of publication April 2, 2014; date of current version June 18, 2014. This work was supported by the U.S. National Science Foundation under Grant CNS-1110947. The associate editor coordinating the review of this paper and approving it for publication was O. B. Akan.

M. Pierobon is with the Department of Computer Science \& Engineering, University of Nebraska-Lincoln, Lincoln, NE 68588 USA (e-mail: pierobon@ cse.unl.edu).

I. F. Akyildiz is with the Broadband Wireless Networking Laboratory, School of Electrical and Computer Engineering, Georgia Institute of Technology, Atlanta, GA 30332 USA (e-mail: ian@ece.gatech.edu).

Color versions of one or more of the figures in this paper are available online at http://ieeexplore.ieee.org.

Digital Object Identifier 10.1109/TCOMM.2014.2314650 natural communications in biology, is expected to be especially attractive due to its inherent biocompatibility. Envisioned applications [2] of molecular nanonetworks range from the biomedical field, in intra-body diagnosis and intelligent drug delivery, to the industrial fields, as a support to the monitoring and control of goods production and waste disposal, and security/ safety applications, such as for biological and chemical attack detection.

Several MC techniques have been proposed so far, which differentiate themselves for the way in which molecules are propagated from the transmitter to the destination device. These techniques involve either passive molecule transport (diffusionbased architectures [3]) or active molecule transport (molecular motors [4], bacteria chemotaxis [5]). We consider the diffusionbased architectures as the most general alternative, since they do not rely on an ad-hoc infrastructure for molecule propagation, but they are based on the spontaneous Brownian motion. We believe that by studying the diffusion-based MC we can provide solutions that can then be easily tailored and expanded to be applied to other more specific MC techniques.

Molecular nanonetworks architectures based on diffusionbased MC have been a subject of study in the latest years and contributions from the literature propose diverse solutions on the way to encode information in the diffusing molecules. In [6] the information is encoded in the time of arrival of molecules at the receiver, in contrast to [7] where each molecule carries a piece of information according to its molecular composition. Other contributions [8]-[14] agree on the higher potential of encoding the information in the variations of the concentration of molecules in the space, which is also confirmed by our studies [15] on the information capacity of this architecture.

In this paper, we focus on the study of the interference in diffusion-based molecular nanonetworks by taking into account the information encoding on the molecule concentration. Previous literature has addressed the problem of diffusionbased MC interference in the same architecture. In [16] the effects of intersymbol and co-channel interference are analyzed in reference to two specific modulation techniques proposed by the same authors. In [17] the intersymbol interference is characterized in a unicast MC system with binary amplitude modulation. In [18], interference is studied for another specific modulation technique, based on the transmission order of different types of molecules. Our contribution in [19] characterizes the intersymbol and co-channel interference by modeling the attenuation and dispersion of Gaussian pulses. The interference analysis provided in this paper completes our work on the end-to-end physical and noise modeling of diffusion-based MC published in [12]-[14], respectively, by providing closed-form 
mathematical expressions to evaluate the impact of interference in an MC system.

The objective of this paper is to provide a statistical-physical modeling of the interference in the diffusion-based molecular nanonetworks when multiple transmitting nanomachines emit molecules simultaneously. Since our goal is to consider a worst case scenario for the analysis of interference, we do not consider coordination among transmitting nanomachines, whose emissions at any time instant are random and modeled through a stochastic process. In diffusion-based molecular nanonetworks, interference can be considered either as a disturbance in the reception of one or more molecular signals, or as an effect that can be exploited to enhance the communication of information from many/all transmitting nanomachines. As an example, nanomachines could send the same molecular signal upon sensing an over-threshold value in the environment. The interference of these signals at the receiver could then convey information regarding not only the presence of an overthreshold value, but also the number of transmitters that are detecting this value. The results presented in this paper will support the end-to-end design of these systems by providing a mathematical framework to quantify the impact of interference in diffusion-based MC as function of physical parameters. Our method to characterize interference differentiates from the previous literature on molecular nanonetworks, since we develop a general model independent from specific modulation and coding techniques. In particular, this modeling stems from the same general assumptions used in interference study for radio communication networks, namely, a spatial Poisson distribution of interfering transmitters having independent and identically distributed (i.i.d.) emissions. Moreover, the specific probability distribution used for the molecule emissions is in agreement with a chemical description of the transmitters in terms of Langevin equation [20], which models the randomness in the chemical reactions involved in the production of the molecules.

The statistical-physical modeling detailed in this paper is based on the property of the received molecular signal of being a stationary Gaussian Process (GP), which results from the molecule emission distribution and the diffusionbased molecule propagation. As a consequence, the statisticalphysical modeling is operated on the received Power Spectral Density (PSD), for which it is possible to obtain an analytical expression of the log-characteristic function. The expression of the received PSD log-characteristic function ultimately leads to the estimation of the received PSD probability distribution. The received PSD probability distribution provides a complete description of the GP of the received molecular signal, which corresponds to the interference in diffusion-based molecular nanonetworks. By using the derived statistical-physical interference model, we also provide numerical results in terms of received PSD probability distribution and probability of interference for selected values of the physical parameters of the molecular nanonetwork, such as the diffusion coefficient, the transmitter density and the average power of molecule emissions, and we compare them with the outcomes of a simulation environment.

The remainder of the paper is organized as follows. In Section II we list the main reference models, assumptions, and

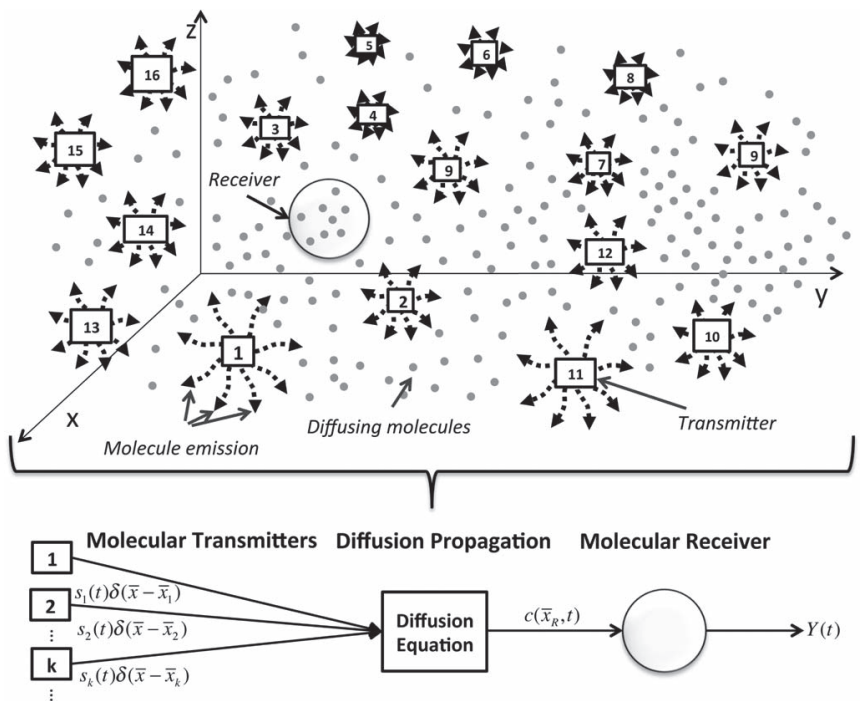

Fig. 1. Reference diffusion-based molecular nanonetwork considered for the interference modeling.

definitions used in this paper for the statistical-physical modeling of the interference in molecular nanonetworks. The main goals of the statistical-physical modeling of the interference are introduced in Section III, together with the probabilistic description of the received signal and a description of the steps for the statistical-physical modeling of the received PSD. In Section IV, we analytically compute the log-characteristic function of the received PSD and numerically derive its PDF. Numerical results are provided in Section V for the simulationbased evaluation and the probability of interference. Finally, in Section VI, we conclude the paper.

\section{INTERFERENCE IN MOLECULAR NANONETWORKS}

In this section, we describe the main reference models, assumptions, and definitions used in this paper for the statistical-physical modeling of the interference in molecular nanonetworks.

\section{A. Reference Molecular Nanonetwork}

In the following, we detail the main elements of the reference molecular nanonetwork considered in this paper. As sketched in Fig. 1, these elements are the molecular transmitters, responsible for the emission of molecular signals, the diffusion-based propagation, which broadcasts the molecular signals in the space by means of free molecule diffusion, and the molecular receiver, which senses the incoming molecular signals.

A Molecular Transmitter, identified by a number $k$ and located at $\bar{x}_{k}$, is responsible for the emission of molecules in the space according to a molecular signal $s_{k}(t)$ as function of the time $t$. In general, the molecular signal $s_{k}(t)$ can assume any value as function of the time $t$, provided that the concentration of molecules in the space is always kept at a positive value, as explained in the following. We assume that all the transmitters emit molecules of the same species $n$ within an equal definite volume $V_{T}$, whose size is negligible with respect to the distance 
between each transmitter and the receiver. ${ }^{1}$ Upon this emission of molecules, identified with the time derivative $d X_{n}(t) / d t$ in the number $X_{n}$ of molecules of species $n$ inside the volume $V_{T}$ at the transmitter $k$, each molecular transmitter $k$ causes a change in the molecule concentration $c(\bar{x}, t)$ at its location $\bar{x}_{k}$, which is expressed through the following relation:

$$
\frac{\partial c(\bar{x}, t)}{\partial t}=\frac{1}{V_{T}} \frac{d X_{n}(t)}{d t} \delta\left(\bar{x}-\bar{x}_{k}\right)=s_{k}(t) \delta\left(\bar{x}-\bar{x}_{k}\right)
$$

where $\partial c(\bar{x}, t) / \partial t$ is the time derivative in the molecule concentration at the location $\bar{x}$ and time $t$, and $\delta($.$) is the Dirac$ delta. Moreover, we assume that a transmitter is able to produce molecules, thus resulting in a positive time first derivative $d X_{n}(t) / d t>0$ and a positive transmitted signal $s_{k}(t)>0$, or to subtract molecules, thus resulting in a negative time first derivative $d X_{n}(t) / d t<0$ and in a negative transmitted signal $s_{k}(t)<0$. These processes are identified in Section II-B with chemical reactions, where the molecules composing the molecular signal can appear as either products or reactants. The former case corresponds to the production of these molecules at the transmitter location, while the latter case corresponds to the alteration of these molecules into a form that cannot be recognized by the molecular receiver, and it is equivalent to subtracting these molecules from the space at the transmitter location.

The Diffusion Propagation broadcasts the emitted molecular signal $s_{k}(t)$ from each transmitter location $\bar{x}_{k}$ to any other location $\bar{x}$ in the space. In this paper, we rely on the assumption to have a 3-d space, which contains a fluidic medium and has infinite extent in all the three dimensions. Moreover, the molecules of species $n$ are all identical and undistinguishable, and they move independently from each other according to the Brownian motion. We define the total molecule concentration in the space as $c_{\text {base }}+c(\bar{x}, t)$, where $c_{\text {base }}$ is a component of the molecule concentration that is positive, homogeneous in the space, and constant in time, while $c(\bar{x}, t)$ is the varying component of the molecule concentration as a function of the space $\bar{x}$ and time $t$. We assume that the component $c_{\text {base }}$ has a value sufficient to keep the total molecule concentration positive throughout the space even when $c(\bar{x}, t)<0$ due to transmitters subtracting molecules from the space, $s_{k}(t)<0$. Consequently, the value of $c_{b a s e}$ is chosen according to the molecular signals $s_{k}(t)$ that we intend to transmit. The diffusion propagation is based on the following Diffusion Equation [23], [24] in the variable $c(\bar{x}, t)$ :

$$
\frac{\partial c(\bar{x}, t)}{\partial t}=D \nabla^{2} c(\bar{x}, t)
$$

\footnotetext{
${ }^{1}$ As an example, we consider the size of a molecular transmitter or receiver within the same order of magnitude of the size of the components used in nature by cells to realize information molecule exchange via chemical reactions (biological circuits) [21], whose mathematical model is presented in Section II-B. These components can be identified with DNA strands (whose average size ranges from a fraction to tens of $\mathrm{nm}$ ), which contain the necessary information to encode enzymes to produce cellular signaling molecules or chemical receptors for signaling molecule concentration detection [21], RNA polymerases (sized around $15 \mathrm{~nm}$ ), which realize the DNA transcription process, and ribosomes (with average size of tens of $\mathrm{nm}$ ), which realize the DNA translation process [22]. Overall, we can consider the minimum size of the transmitter volume $V_{T}$ as being in the order of tens of $\mathrm{nm}$.
}

where $\partial(.) / \partial t$ and $\nabla^{2}($.$) are the time first derivative and the$ Laplacian operator (sum of the 3-d spatial second derivatives), respectively. $D$ is the diffusion coefficient and it quantifies the total flux of molecules, equal to a molecule concentration moving with a definite velocity in the three spatial coordinates, generated by diffusion in response to a gradient of the molecule concentration in the space, equal to the first derivative of the molecule concentration with respect to the three spatial coordinates. The diffusion coefficient $D$ is considered a scalar (the molecule flux in response to a concentration gradient is equal in all three spatial coordinates), constant in time, and homogeneous in the space within the scope of this paper, and this is in agreement with the assumption of having independent Brownian motion for every molecule in the space [23], [24]. As a consequence of this assumption, the diffusion coefficient $D$ depends on the absolute temperature of the system $T$, assumed constant and homogeneous, the molecule shape and size, and the fluid viscosity $\eta$, which quantifies the resistance of the fluid to the molecule motion. If molecules are approximated by spherical particles with radius $\rho_{p}$, then the diffusion coefficient $D$ has the following expression:

$$
D=\frac{k_{B} T}{6 \pi \eta \rho_{p}}
$$

where $k_{B}$ is the Boltzmann's constant. Typical values of the diffusion coefficient $D$ at room temperature for common elements (e.g., oxygen) in air are around $0.2\left[\mathrm{~cm}^{2} \mathrm{sec}^{-1}\right]$, while in water are around $10^{-5}\left[\mathrm{~cm}^{2} \mathrm{sec}^{-1}\right]$ [24]. The diffusion of molecules in a biological environment, characterized by a higher viscosity with respect to water, has a value around $10^{-6}\left[\mathrm{~cm}^{2} \mathrm{sec}^{-1}\right]$ (cellular cytoplasm, [25]).

The Molecular Receiver senses the total incoming molecular concentration $c_{\text {base }}+c\left(\bar{x}_{R}, t\right)$ at its location $\bar{x}_{R}$ and recovers the received signal $Y(t)$ from the varying component $c\left(\bar{x}_{R}, t\right)$. This is expressed by the following relation:

$$
Y(t)=c\left(\bar{x}_{R}, t\right) .
$$

As a consequence, when no transmitter is emitting molecular signals (creating or subtracting molecules), the total molecule concentration is constant and equal to $c_{b a s e}$, and the received signal $Y(t)$ is equal to zero. The molecular receiver is considered in this paper as an ideal molecular concentration detector located at $\bar{x}_{R}$. This assumption is in agreement with the goal of this work of analyzing the interference of multiple transmitted signals at a location in the space, and it is an approximation of a real molecular receiver whose size is negligible with respect to the distance to each considered molecular transmitter.

\section{B. Assumptions on Interferers}

For our interference study, we consider multiple molecular transmitters (interferers), each one emitting a molecular signal from a different location. We apply the following assumptions:

- The molecular transmitters are assumed to be infinite in number and distributed in the 3-d space according to a 
spatial homogeneous Poisson process ${ }^{2}$ whose rate is equal to the transmitter density $\lambda$, which corresponds to the average number of transmitters per unit volume. For this, the probability to find a number $K$ of transmitters in a region $V$ of the space is expressed as follows:

$$
P(K \text { transmitters in } V)=\frac{[\lambda V]^{K} e^{-\lambda V}}{K !} .
$$

- The molecular transmitters emit independent and identically distributed (i.i.d.) molecular signals $s_{k}(t)$. Each $s_{k}(t)$ is a white Gaussian signal [28], whose values at each time instant $t$ have zero mean and variance equal to $\sigma^{2}$, expressed as

$$
s_{k}(t) \sim \mathcal{N}\left(0, \sigma^{2}\right) \quad \forall t
$$

The expression in (6) models the variability of the transmitter emissions according to the variance parameter $\sigma^{2}$. As demonstrated in Appendix A, the parameter $\sigma^{2}$ corresponds to the average power, or variance, of the rate of produced molecules per unit time from the chemical reactions occurring inside the receiver volumes when at equilibrium. Consequently, $\sigma^{2}$ quantifies the variability of the signals emitted by different molecular transmitters.

\section{Definition of Interference}

We define as interference the received signal $Y(t)$ expressed as the propagation function $f_{d}($.$) of the multiple transmitted$ molecular signals $s_{k}$, where $k=0, \ldots, \infty$, as follows:

$$
Y(t)=f_{d}\left(\sum_{k=0}^{\infty} s_{k}(t) \delta\left(\bar{x}-\bar{x}_{k}\right)\right)
$$

where $\delta($.$) is the Dirac delta, f_{d}($.$) is the diffusion propaga-$ tion function that transforms the sum of transmitted molecular signals $s_{k}$ into the incoming molecular concentration $c\left(\bar{x}_{R}, t\right)$ at the receiver location $\bar{x}_{R}$ through the diffusion (2) and, according to (4), into the received signal $Y(t)$.

Due to the linearity of (2) [23], [24], given multiple molecular signals transmitted simultaneously from multiple transmitters, the resulting varying component $c(\bar{x}, t)$ of the molecule concentration is the sum of the varying components of the molecule concentration resulting from the emission of each molecular transmitter, computed as if each transmitter were emitting alone (additive channel). As a consequence, we can express the received signal $Y(t)$ as the sum of the propagation functions applied separately to each transmitted molecular signal, which results into

$$
Y(t)=\sum_{k=0}^{\infty} f_{d}\left(s_{k}(t) \delta\left(\bar{x}-\bar{x}_{k}\right)\right)
$$

\footnotetext{
${ }^{2}$ The use of a spatial Poisson process to model the molecular transmitter distribution is in agreement with the stochastic modeling in biology of the spatial distribution of cells or cell groups, which exchange information through molecular communication. As an example, bacteria in a plate or bacteria colonies in a 3-d medium, such as cheese [26], are proven to have a distribution that fits a Poisson process [27].
}

where the propagation function $f_{d}($.$) is computed as the so-$ lution of the diffusion equation (2) when a single transmitter is emitting a molecular signal $s_{k}(t)$. For this, we consider the following expression:

$$
\frac{\partial c(\bar{x}, t)}{\partial t}=D \nabla^{2} c(\bar{x}, t)+s_{k}(t) \delta\left(\bar{x}-\bar{x}_{k}\right) .
$$

The solution of (9) in terms of $c(\bar{x}, t)$ corresponds to the following propagation function $f_{d}($.$) :$

$$
\begin{aligned}
f_{d}\left(s_{k}(t) \delta\left(\bar{x}-\bar{x}_{k}\right)\right) & =c(\bar{x}, t) \\
& =g_{d}\left(r_{k}, t\right) * s_{k}(t) \\
& =\int_{0}^{\infty} g_{d}\left(r_{k}, \tau\right) s_{k}(\tau-t) d \tau
\end{aligned}
$$

where $(. *$.$) is the convolution operator [29], and g_{d}\left(r_{k}, t\right)$ is the Green's function of the diffusion equation [30], equal to

$$
g_{d}\left(r_{k}, t\right)=\frac{e^{-\frac{r_{k}{ }^{2}}{4 D t}}}{(4 \pi D t)^{3 / 2}}
$$

where $r_{k}$ is the Euclidian distance between the transmitter $k$ location and the receiver location, $r_{k}=\left|\bar{x}_{k}-\bar{x}_{R}\right|$, and $D$ is the diffusion coefficient. As a result, we can express the received signal $Y(t)$ as

$$
Y(t)=\sum_{k=0}^{\infty} g_{d}\left(r_{k}, t\right) * s_{k}(t)
$$

where $g_{d}\left(r_{k}, t\right)$ is expressed in $(11),(. *$.$) is the convolution$ operator [29], and $s_{k}(t)$ is the molecular signal transmitted from each transmitter $k$, whose distribution is given by (6).

\section{Statistical-Physical Interference Modeling}

The goal of the statistical-physical interference modeling is to find a probabilistic description of the received signal $Y(t)$ expressed in (12), as function of the transmitter density $\lambda$, the diffusion coefficient $D$, and the average power $\sigma^{2}$ of the molecular signals emitted by the transmitters.

In standard statistical-physical modeling of the interference for radio communication networks [31], since the propagation function corresponds to a multiplication of each transmitted signal (uncorrelated random process with zero mean value) by the radio propagation amplitude loss, independent with respect to the time variable, the received signal $Y(t)$ is an uncorrelated stochastic process with zero mean value. As a consequence, the received signal $Y(t)$ can be probabilistically described with the Probability Density Function (PDF) $P_{Y}(y)$ of a time sample, for which analytical expressions are usually provided in terms of log-characteristic functions [32].

\section{A. Probabilistic Description of the Received Signal}

In the context of diffusion-based molecular nanonetworks, as a consequence of the expression of the propagation function in (11) as function of the time variable $t$, the received signal 
$Y(t)$ is in general a correlated stochastic process, which cannot be described by the PDF $P_{Y}(y)$ of a single time sample. A probabilistic description of the received signal $Y(t)$ can be provided upon the following considerations:

- Consider a realization of the spatial homogeneous Poisson process of the transmitter locations, expressed in (5), which results into a set of values $R=\left\{r_{k}\right\}_{k=1,2, \ldots, \infty}$ for the distances $r_{k}$ between each transmitter $k=1,2 \ldots, \infty$ and the receiver.

- Given the previous consideration, each term of the sum in (12) is a convolution of a deterministic function $g_{d}\left(r_{k}, t\right)$ of the time $t$ with a zero-mean Gaussian white random signal $s_{k}(t)$ with zero mean and variance equal to $\sigma^{2}$. The result of this convolution is a zero-mean stationary Gaussian process $y_{k} \mid r_{k}$ with autocorrelation function $R_{y_{k} \mid r_{k}}(t)$ equal to $\sigma^{2}$ multiplied by the correlation of $g_{d}\left(r_{k}, t\right)$ with itself [28]. This is expressed as follows:

$$
\mathcal{R}_{y_{k} \mid r_{k}}(t)=\sigma^{2} \int_{0}^{\infty} g_{d}\left(r_{k}, \tau\right) g_{d}\left(r_{k}, \tau+t\right) d \tau .
$$

- The autocorrelation of the sum of two uncorrelated random processes is a random process whose autocorrelations is the sum of their autocorrelations [28].

As a consequence of the aforementioned considerations, the received signal $Y \mid R$, given a realization of the transmitter locations $R$, is a zero-mean stationary Gaussian Process (GP), probabilistically described as follows:

$$
Y \mid R \sim \mathbf{G P}\left(0, \mathcal{R}_{Y \mid R}(t)\right)
$$

whose autocorrelation function $\mathcal{R}_{Y \mid R}(t)$ is equal to the sum for each transmitter $k=1,2, \ldots, \infty$ of the autocorrelation function $\mathcal{R}_{y_{k} \mid r_{k}}(t)$ in (13), expressed as

$$
\mathcal{R}_{Y \mid R}(t)=\sum_{k=0}^{\infty} \mathcal{R}_{y_{k} \mid r_{k}}(t)
$$

Since $Y \mid R$ is a continuous time stationary random process, according to the Wiener-Khintchine theorem [28] it can be equivalently described in terms of Power Spectral Density (PSD), which corresponds to the Fourier transform [29] of the autocorrelation function $\mathcal{R}_{Y \mid R}(t)$. Given the expressions in (15) and (13), the PSD $\mathcal{S}_{Y \mid R}(\omega)$ results in the following:

$$
\mathcal{S}_{Y \mid R}(\omega)=\sigma^{2} \sum_{k=0}^{\infty}\left|G_{d}\left(r_{k}, \omega\right)\right|^{2}
$$

where $|.|^{2}$ denotes the squared absolute value operator, and $G_{d}\left(r_{k}, \omega\right)$ is the Fourier transform [29] of $g_{d}\left(r_{k}, t\right)$ in (11), whose expression is

$$
G_{d}\left(r_{k}, \omega\right)=\frac{e^{-(1+j) \sqrt{\frac{\omega}{2 D}} r_{k}}}{\pi D r_{k}}
$$

where $r_{k}$ is the Euclidian distance between the transmitter $k$ and the receiver, and $D$ is the diffusion coefficient.

\section{B. Statistical-Physical Modeling of the Received Power Spectral Density}

The received PSD $\mathcal{S}_{Y}(\omega)$ is defined as the distribution of the power of the received signal $Y$ over each frequency $\omega$. Given the presence of multiple transmitters, and the probabilistic assumptions described in Section II-B, the received PSD $\mathcal{S}_{Y}(\omega)$ is a measure of the power of the interference which affects the communication system in each received frequency $\omega$. As a consequence, we aim at the statistical-physical modeling of the received PSD $\mathcal{S}_{Y}(\omega)$ through the expression of its PDF $P_{\mathcal{S}_{Y}(\omega)}(s)$ as a function $f($.$) of the PSD value s$, the frequency $\omega, \lambda, D$, and $\sigma^{2}$. This is expressed as follows:

$$
P_{\mathcal{S}_{Y}(\omega)}(s)=f\left(s, \omega, \lambda, D, \sigma^{2}\right) .
$$

As detailed in the following, the PDF $P_{\mathcal{S}_{Y}(\omega)}(s)$ of the PSD $\mathcal{S}_{Y}(\omega)$ is computed from the PSD $\mathcal{S}_{Y \mid R}(\omega)$ in (16) by taking into account the spatial homogeneous Poisson process of the transmitter locations in (5).

The PDF $P_{\mathcal{S}_{Y}(\omega)}(s)$, as happens in standard statistical-physical modeling for the PDF $P_{Y}(y)$ of the interference for radio communication networks [31], does not have a closed-form mathematical expression. As a consequence, we aim at the expression of the log-characteristic function $\psi_{\mathcal{S}_{Y}(\omega)}(\Omega)$ of the received PSD $\mathcal{S}_{Y}(\omega)$, which is defined as the natural logarithm of the characteristic function $\phi_{\mathcal{S}_{Y}(\omega)}(\Omega)$, as

$$
\psi_{\mathcal{S}_{Y}(\omega)}(\Omega)=\ln \left[\phi_{\mathcal{S}_{Y}(\omega)}(\Omega)\right]
$$

The characteristic function $\phi_{\mathcal{S}_{Y}(\omega)}(\Omega)$ of the received PSD $\mathcal{S}_{Y}(\omega)$ is defined as the expected value of the function $e^{j \Omega s}$ of the PSD value $s$

$$
\phi_{\mathcal{S}_{Y}(\omega)}(\Omega)=E_{\mathcal{S}_{Y}(\omega)}\left[e^{j \Omega s}\right]=\int P_{\mathcal{S}_{Y}(\omega)}(s) e^{j \Omega s} d s
$$

The PDF $P_{\mathcal{S}_{Y}(\omega)}(s)$ of the PSD $\mathcal{S}_{Y}(\omega)$ is computed through the Fourier transform [29] of the exponential with the logcharacteristic function $\psi_{\mathcal{S}_{Y}(\omega)}(\Omega)$ as argument. This is expressed as follows:

$$
P_{\mathcal{S}_{Y}(\omega)}(s)=\int e^{\psi_{\mathcal{S}_{Y}(\omega)}(\Omega)} e^{-j \Omega y} d \Omega
$$

As mentioned above, the formula in (21) does not in general result in a closed-form expression, and it is computed through numerical methods.

In the following, we derive the log-characteristic function $\psi_{\mathcal{S}_{Y}(\omega)}(\Omega)$, which admits an analytical expression as a function $\Psi($.$) of \lambda, D, \sigma^{2}$, the PSD frequency variable $\omega$, and the characteristic function frequency variable $\Omega$, expressed as follows:

$$
\psi_{\mathcal{S}_{Y}(\omega)}(\Omega)=\Psi\left(\lambda, D, \sigma^{2}, \omega, \Omega\right) .
$$




\section{LOG-ChARACTERISTIC FUnCTION AND PDF OF THE Received Power Spectral Density}

In this section, we analytically derive the log-characteristic function $\psi_{\mathcal{S}_{Y}(\omega)}(\Omega)$ of the received PSD $\mathcal{S}_{Y}(\omega)$. Through the derivation detailed in Section IV-A, we obtain the following analytical expression:

$$
\psi_{\mathcal{S}_{Y}(\omega)}(\Omega)=j \frac{16 \sqrt{2} \lambda \sigma^{2} \Omega}{3 \pi \sqrt{D^{3} \omega}} \int_{0}^{\infty}(x+1) e^{-2 x} e^{-j \frac{e^{-2 x}}{x^{2}} \frac{\sigma^{2} \omega \Omega}{2 \pi^{2} D^{3}}} d x
$$

where $\lambda$ is the transmitter density (number of transmitters per unit volume), $D$ is the diffusion coefficient, $\sigma^{2}$ is the average power of the molecular signals emitted by the transmitters, $\omega$ is the PSD frequency variable, and $\Omega$ is the frequency variable of the characteristic function. Subsequently, we derive the PDF $P_{\mathcal{S}_{Y}(\omega)}(s)$ of the received PSD $\mathcal{S}_{Y}(\omega)$ by numerically computing the expression in (21).

\section{A. Derivation of the Log-Characteristic Function $\psi_{\mathcal{S}_{Y}(\omega)}(\Omega)$}

In the following, we derive the log-characteristic function $\psi_{\mathcal{S}_{Y}(\omega)}(\Omega)$ of the received PSD $\mathcal{S}_{Y}(\omega)$ in diffusion-based molecular nanonetworks. By applying the rule of the iterated expectations [28], we can perform the expectation in (20) with respect to the transmitter locations $R=\left\{r_{k}\right\}_{k=1,2, \ldots, \infty}$, where $r_{k}$ are the random distances between each transmitter $k=$ $1,2, \ldots, \infty$ and the receiver, and substitute the PSD value $s$ with the PSD $\mathcal{S}_{Y \mid R}(\omega)$ of the received signal given a realization of the transmitter locations. As a consequence, we obtain the following expression:

$$
\phi_{\mathcal{S}_{Y}(\omega)}(\Omega)=E_{R}\left[e^{j \Omega \mathcal{S}_{Y \mid R}(\omega)}\right]
$$

where the PSD $\mathcal{S}_{Y \mid R}(\omega)$ is computed through (16) and (17).

Since the transmitter locations are resulting from a spatial homogeneous Poisson process, as described in Section II-B, the distances $r_{k}$ are i.i.d. random variables, and the distribution in the number $k$ of molecular transmitters in a space region $V$ is given by (5). As a consequence, for an infinite space region, represented by a sphere centered at the receiver with infinite radius, namely, $V=\lim _{\rho \rightarrow \infty}(4 / 3) \pi \rho^{3}$, we derive the following expression from (16) applied to (24):

$$
\begin{array}{r}
\phi_{\mathcal{S}_{Y}(\omega)}(\Omega)=\lim _{\rho \rightarrow \infty} \sum_{k=0}^{\infty}\left(E_{r_{k}}\left[e^{j \Omega \sigma^{2}\left|G_{d}\left(r_{k}, \omega\right)\right|^{2}}\right]\right)^{k} \\
\cdot \frac{\left[\lambda(4 / 3) \pi \rho^{3}\right]^{k} e^{-\lambda(4 / 3) \pi \rho^{3}}}{k !}
\end{array}
$$

where the summation from (16) is substituted with the power $k$ operator $(.)^{k}$, and the average operator $E_{R}[$.$] is written in$ terms of summation in $k$ of the average operator $E_{r_{k}}[$.] of the $k$-th transmitter distance, weighted by the probability density from (5).
By applying the following Taylor series expansion [33] substitution to (25):

$$
\sum_{k=0}^{\infty} \frac{x^{k}}{k !}=e^{x}
$$

and by applying the definition of log-characteristic function $\psi_{\mathcal{S}_{Y}(\omega)}(\Omega)$ from (19), we obtain the following expression:

$$
\psi_{\mathcal{S}_{Y}(\omega)}(\Omega)=\lim _{\rho \rightarrow \infty} \frac{4}{3} \pi \rho^{3} \lambda\left(E_{r_{k}}\left[e^{j \Omega \sigma^{2}\left|G_{d}\left(r_{k}, \omega\right)\right|^{2}}\right]-1\right) .
$$

Since the transmitters are distributed according to a Poisson process (5), the distribution of the distance between the transmitter and the receiver, given a space region $V=(4 / 3) \pi \rho^{3}$, has the following expression:

$$
P_{r_{k}}(r)=\frac{3 r^{2}}{\rho^{3}}, \quad 0 \leq r \leq \rho .
$$

If we express in (27) the average operator $E_{r_{k}}[$.$] of the distance$ $r_{k}$ between the transmitter and the receiver by using the distribution of this distance in (28), we obtain the following:

$$
\psi_{\mathcal{S}_{Y}(\omega)}(\Omega)=\lim _{\rho \rightarrow \infty} \frac{4}{3} \pi \rho^{3} \lambda\left(\int_{0}^{\rho} e^{j \Omega \sigma^{2}\left|G_{d}(r, \omega)\right|^{2}} \frac{3 r^{2}}{\rho^{3}} d r-1\right) .
$$

By using the formula of the integration by parts [33] for the integral in (29), we obtain the following expression:

$$
\begin{array}{r}
\psi_{\mathcal{S}_{Y}(\omega)}(\Omega)=\lim _{\rho \rightarrow \infty} \frac{4}{3} \pi \rho^{3} \lambda\left(e^{j \Omega \sigma^{2} \frac{e^{-2} \sqrt{\frac{\omega}{2 D}} \rho}{(\pi D \rho)^{2}}}+-\frac{4 j \Omega \sigma^{2}}{\rho^{3}(\pi D)^{2}}\right. \\
\left.\times \int_{0}^{\rho}\left(\sqrt{\frac{\omega}{2 D}} r+1\right) \cdot e^{-2 \sqrt{\frac{\omega}{2 D}} r} e^{j \Omega \sigma^{2} \frac{e^{-2} \sqrt{\frac{\omega}{2 D}} r}{(\pi D r)^{2}}} d r-1\right) .
\end{array}
$$

We note the following result:

$$
\lim _{\rho \rightarrow \infty} \rho^{3}\left(e^{j \Omega \sigma^{2} \frac{e^{-2} \sqrt{\frac{\omega}{2 D}} \rho}{(\pi D \rho)^{2}}}-1\right)=0
$$

which is demonstrated by considering the following inequality:

$$
\frac{e^{-2 \sqrt{\frac{\omega}{2 D}} \rho}}{(\pi D \rho)^{2}}<\frac{1}{\rho}, \quad \text { for } \rho \rightarrow \infty
$$

and by repeatedly applying L'Hôpital's rule [33] to the following limit:

$$
\lim _{\rho \rightarrow \infty} \rho^{3}\left(e^{1 / \rho}-1\right)=0 .
$$

By applying (31) to (30), we obtain the following expression:

$$
\begin{aligned}
\psi_{\mathcal{S}_{Y}(\omega)}(\Omega)=j \frac{16}{3} \frac{\lambda \sigma^{2} \Omega}{\pi D^{2}} \int_{0}^{\infty}\left(\sqrt{\frac{\omega}{2 D}} r+1\right) \\
\cdot e^{-2 \sqrt{\frac{\omega}{2 D}} r} e^{j \Omega \sigma^{2} \frac{e^{-2} \sqrt{\frac{\omega}{2 D}} r}{(\pi D r)^{2}}} \frac{r^{3}}{R^{3}} d r .
\end{aligned}
$$




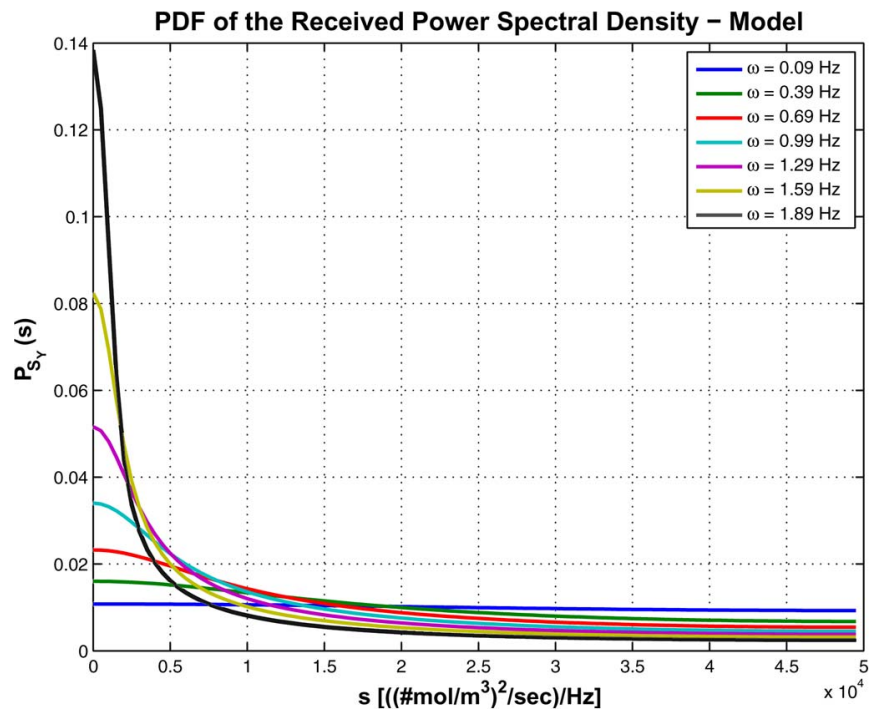

Fig. 2. PDF $P_{\mathcal{S}_{Y}(\omega)}(s)$ of the received PSD $\mathcal{S}_{Y}(\omega)$. Different curves refer to different values of the frequency $\omega$.

By operating in the integral of (34) the following variable substitution:

$$
x=\sqrt{\frac{\omega}{2 D}} r
$$

we obtain the final expression of the log-characteristic function $\psi_{\mathcal{S}_{Y}(\omega)}(\Omega)$ of the received PSD $\mathcal{S}_{Y}(\omega)$, which is as follows:

$$
\psi_{\mathcal{S}_{Y}(\omega)}(\Omega)=j \frac{16 \sqrt{2} \lambda \sigma^{2} \Omega}{3 \pi \sqrt{D^{3} \omega}} \int_{0}^{\infty}(x+1) e^{-2 x} e^{-j \frac{e^{-2 x}}{x^{2}} \frac{\sigma^{2} \omega \Omega}{2 \pi^{2} D^{3}}} d x .
$$

\section{B. Derivation of the PDF $P_{\mathcal{S}_{Y}(\omega)}(s)$}

In this section, we derive the $\operatorname{PDF} P_{\mathcal{S}_{Y}(\omega)}(s)$ of the received PSD $\mathcal{S}_{Y}(\omega)$. In general, the log-characteristic function expressed in (36) does not have an expression which can be recognized as from a known probability distribution. For this, we numerically compute the formula in (21) by using the MATLAB fft function applied to the values of the expression in (36). We also numerically compute the infinite integral in (36) by using the MATLAB numerical integration.

The numerical results in terms of PDF $P_{\mathcal{S}_{Y}(\omega)}(s)$ of the received PSD $\mathcal{S}_{Y}(\omega)$ are shown in Figs. 2 and 3. The values of the PDF $P_{\mathcal{S}_{Y}(\omega)}(s)$ are computed for a transmitter density $\lambda$ equal to $10^{9}$ [transmitters $\mathrm{m}^{-3}$ ], an average power $\sigma^{2}$ of the molecular signals equal to $10^{6}$ [molecules ${ }^{2} \mathrm{~m}^{-6} \mathrm{sec}^{-3}$ ], and for values of the PSD value $s$ ranging from 0 to 5 . $10^{4}\left[\right.$ molecules $^{3} \mathrm{~m}^{-6} \mathrm{sec} \mathrm{Hz}^{-1}$ ]. The diffusion coefficient $D \sim$ $10^{-6}\left[\mathrm{~cm}^{2} \mathrm{sec}^{-1}\right]$ is set to the diffusion coefficient of molecules diffusing in a biological environment (cellular cytoplasm, [25]). Different curves in Fig. 2 refer to different values of the frequency $\omega$, from $0.09 \mathrm{~Hz}$ to $1.89 \mathrm{~Hz}$, while Fig. 3 shows the PDF $P_{\mathcal{S}_{Y}(\omega)}(s)$ values for a range of frequencies $\omega$ from 0 to $2 \mathrm{~Hz}$. This frequency range covers the frequencies of most of the biochemical oscillation mechanisms described in [34],

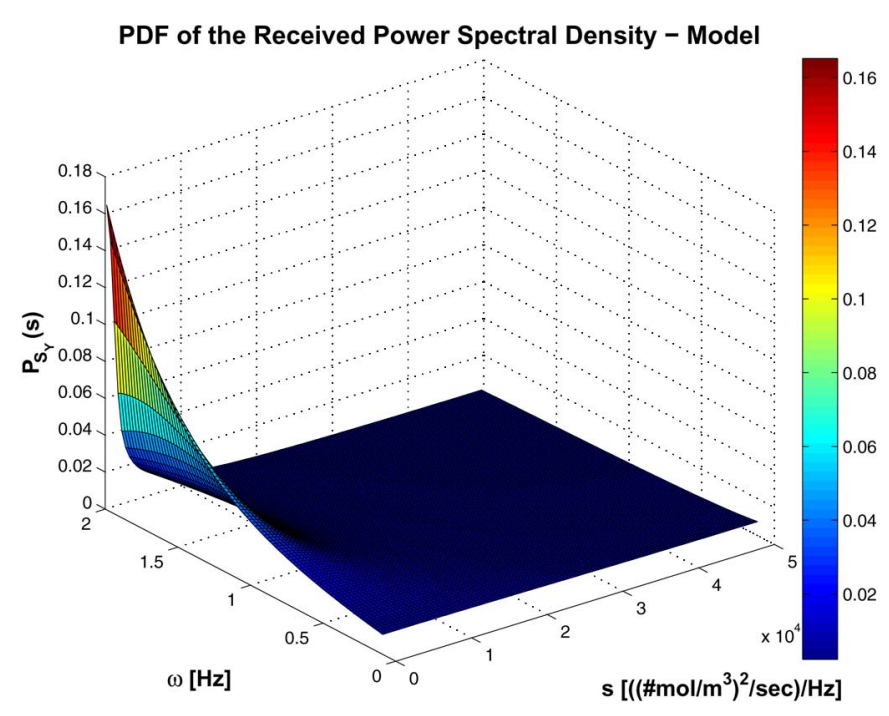

Fig. 3. PDF $P_{\mathcal{S}_{Y}(\omega)}(s)$ of the received PSD $\mathcal{S}_{Y}(\omega)$ for a range of frequencies $\omega$ from 0 to $2 \mathrm{~Hz}$.

including the slower oscillations in neurons that generate the delta waves [35].

As apparent from Figs. 2 and 3, the curves of the PDF $P_{\mathcal{S}_{Y}(\omega)}(s)$ as function of the PSD value $s$ tend to horizontal lines for low values of the frequency $\omega$, while they tend to concentrate the higher values around $s=0$ as the frequency $\omega$ increases. This is an expected behavior since, according to the absolute value of the expression of the Fourier transform of the propagation function $G_{d}\left(r_{k}, \omega\right)$ in (17), which is a negative exponential function of the square root of the frequency $\omega$, lower frequencies are subject to lower attenuation than higher frequencies in the diffusion propagation. As a consequence, for lower frequencies the received PSD tends to have a shape similar to the PSD of the white transmitted signals $s_{k}(t)$ in (6), equally distributed among all the possible PSD values $s$ with a probability value around 0.01 . On the contrary, since higher frequencies are more attenuated, for a high $\omega$ lower values of the received PSD are more probable, which is more likely distributed around $s=0$, with the highest value around 0.16 for $\omega$ close to $2 \mathrm{~Hz}$.

\section{Numerical Results}

In this section, we provide a simulation environment to evaluate the statistical-physical interference model presented in this paper (Section V-A). In addition, we study the probability of interference, defined as the probability for a single molecular signal sent by a transmitter to suffer interference at the receiver, by using both the statistical-physical interference model and the simulation environment (Section V-B).

\section{A. Simulation-Based Evaluation}

The simulation environment is based on the following additional assumptions:

- The space where the transmitters are distributed is confined within a sphere with radius $\rho$ around the receiver location. This is motivated by the need to have in the 
simulation environment a finite number of transmitters, which is equal to $K=\left\lfloor\lambda(4 / 3) \pi \rho^{3}\right\rfloor$, where $\lfloor$.$\rfloor denotes$ the rounding to the nearest lower integer.

- The transmitted signal $s_{k}\left(n / f_{s}\right)$ from each transmitter is discrete, sampled with a frequency $f_{s}$, and composed by $N_{s}$ samples.

- The simulation is repeated for a number of iterations Iter, where each iteration is based on i) a different realization of the spatial Poisson process with density $\lambda$ of the molecular transmitter distribution expressed in (5), ii) a different realization of the Gaussian process in (6) with variance equal to $\sigma^{2}$ for each transmitter $k$ and for each sample $s_{k}\left(n / f_{s}\right)$.

The PDF $P_{\mathcal{S}_{Y}(\omega)}(s)$ of the received PSD $\mathcal{S}_{Y}(\omega)$, where $\omega=$ $q f_{s} / N_{s}$, and $q=1, \ldots, N_{s}$, is computed though the following expression:

$$
\left.P_{\mathcal{S}_{Y}(\omega)}(s)\right|_{\omega=q f_{s} / N_{s}}=\frac{1}{\text { Iter }} \sum_{l=1}^{\text {Iter }} \mathbf{1}_{\mathcal{S}_{Y_{l}}\left(q f_{s} / N_{s}\right)=s}
$$

where $\mathbf{1}_{S_{Y_{i}}\left(q f_{s} / N_{s}\right)=s}$ is non-zero and equal to 1 only when the PSD $S_{Y_{l}}\left(q f_{s} / N_{s}\right)$ from the $l$-th iteration is equal to the value $s$ at frequency $q f_{s} / N_{s}$, and $f_{s}$ and $N_{s}$ are the sampling frequency and the number of samples for the transmitted molecular signals, respectively. The PSD $\mathcal{S}_{Y_{l}}\left(q f_{s} / N_{s}\right)$ results from the following formula:

$$
\mathcal{S}_{Y_{l}}\left(q f_{s} / N_{s}\right)=\left(\sum_{k=1}^{K} S_{k}\left(q f_{s} / N_{s}\right) G_{d}\left(r_{k}, q f_{s} / N_{s}\right)\right)^{2}
$$

where $S_{k}\left(q f_{s} / N_{s}\right)$ is the discrete Fourier transform of $s_{k}\left(n / f_{s}\right)$, computed through the MATLAB $\mathrm{fft}$ function, and $G_{d}\left(r_{k}, q f_{s} / N_{s}\right)$ is the Fourier transform of the propagation function in (17) computed at the frequency value $q f_{s} / N_{s}$.

In Fig. 4 we show the values of $P_{\mathcal{S}_{Y}(\omega)}(s)$ computed for the same parameters as for the results in Fig. 3, namely, a transmitter density $\lambda$ equal to $10^{9}$ [transmitters $\mathrm{m}^{-3}$ ], an average power $\sigma^{2}$ of the molecular signals equal to $10^{6}$ [molecules ${ }^{2} \mathrm{~m}^{-6} \mathrm{sec}^{-3}$ ] a diffusion coefficient $D \sim$ $10^{-6}\left[\mathrm{~cm}^{2} \mathrm{sec}^{-1}\right]$, and for PSD values $s$ ranging from 0 to $5 \cdot 10^{4}$ [molecules ${ }^{3} \mathrm{~m}^{-6} \mathrm{sec} \mathrm{Hz}^{-1}$ ]. Moreover, the simulation is run with the following parameters: a spherical space radius $\rho=19 \mu \mathrm{m}$, a sampling frequency $f_{s}=100 \mathrm{~Hz}$, a number of samples $N_{s}=10^{4}$, and a number of iterations Iter $=50$. The curves in Fig. 4 have been also post-processed through the use of a moving average filter [36] along the dimension of the PSD value $s$ to reduce the noise given by the limited dataset.

The simulation-based results in terms of $P_{\mathcal{S}_{Y}(\omega)}(s)$ in Fig. 4 show a high degree of similarity with the values computed through the statistical-physical model in Fig. 3. Also in the simulation-based results, the curves of $P_{\mathcal{S}_{Y}(\omega)}(s)$ as function of the PSD value $s$ tend to horizontal lines for low values of the frequency $\omega=q f_{s} / N_{s}$, while they tend to concentrate the higher values around $s=0$ as the frequency $\omega$ increases. While for high frequencies $\omega$ around $2 \mathrm{~Hz}$ the simulation-based PDF has a value around $s=0$ of 0.16 , very close to the results of the statistical-physical model, for lower frequencies the values of the model-based PDF are overall lower than the values from

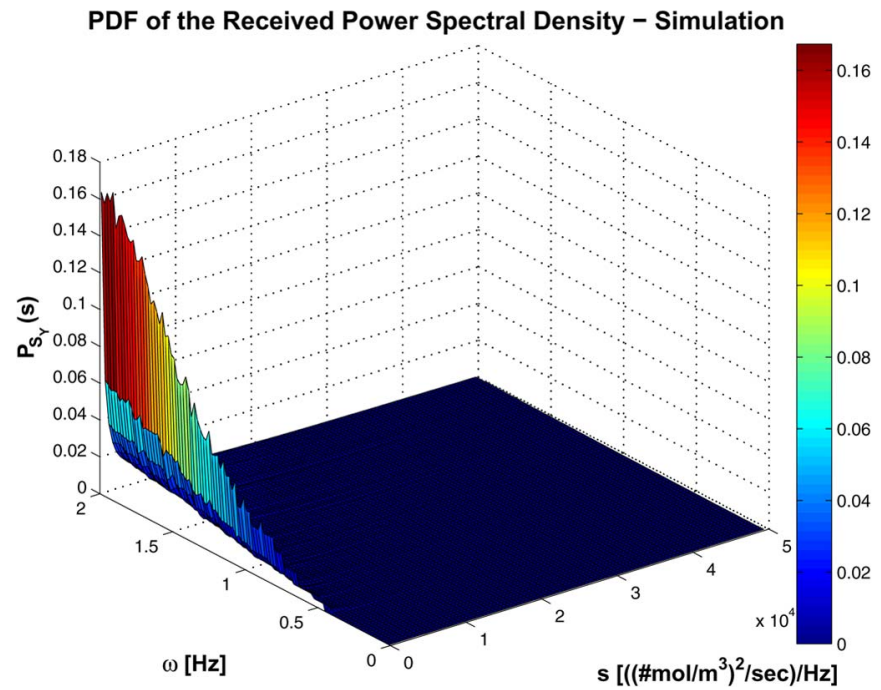

Fig. 4. Simulation-based PDF $P_{\mathcal{S}_{Y}(\omega)}(s)$ of the received PSD $\mathcal{S}_{Y}(\omega)$ for a range of frequencies $\omega$ from 0 to $2 \mathrm{~Hz}$.

the statistical-physical model. We believe that these differences between the values in Fig. 3 and Fig. 4 are due to the limited number of transmitters and the sampling of the molecular signals $s_{k}$ considered for the simulation environment.

\section{B. Probability of Interference}

We define here the probability of interference $P_{\text {Interf }}(\omega)$ as the probability of having at the receiver a contribution from the interference whose PSD at frequency $\omega$ exceeds the PSD of a contribution coming from a single transmitter. This single transmitter is placed at a distance $r_{T x}$ from the receiver, and it transmits a signal $s_{T x}(t)$ with power equal to $\sigma_{t x}^{2}$, expressed as

$$
s_{T x}(t)=\sigma_{t x} \frac{\sin \left[t\left(\omega_{b}-\omega_{a}\right)\right]}{t} e^{j \omega_{a} t} .
$$

The PSD of the signal $s_{T x}(t)$ is then constant over the frequency range defined by $\omega_{a}$ and $\omega_{b}$, and it is expressed as follows:

$$
\mathcal{S}_{T x}(\omega)=\sigma_{t x}^{2} \operatorname{rect}\left(\frac{\omega-\omega_{a}}{\omega_{b}-\omega_{a}}\right)
$$

where rect(.) is the rectangular function, and $\sigma_{t x}^{2}$ is the constant PSD value. The contribution $\mathcal{S}_{R x}(\omega)$ to the PSD of the received signal coming from the transmitted signal $s_{T x}(t)$ is given as

$$
\mathcal{S}_{R x}(\omega)=\mathcal{S}_{T x}(\omega)\left|G_{d}\left(r_{T x}, \omega\right)\right|^{2}
$$

where $G_{d}\left(r_{T x}, \omega\right)$ is the Fourier transform [29] of the Green's function of the diffusion equation expressed in (17). The probability of interference $P_{\text {Interf }}(\omega)$ is expressed as follows:

$$
P_{\text {Interf }}(\omega)=\int_{\mathcal{S}_{R x}(\omega)}^{\infty} P_{\mathcal{S}_{Y}(\omega)}(s) d s
$$

where $\mathcal{S}_{R x}(\omega)$ is the PSD of the signal $s_{T x}(t)$ emitted by the single transmitter, given in (40), and $P_{\mathcal{S}_{Y}(\omega)}(s)$ is the PSD 


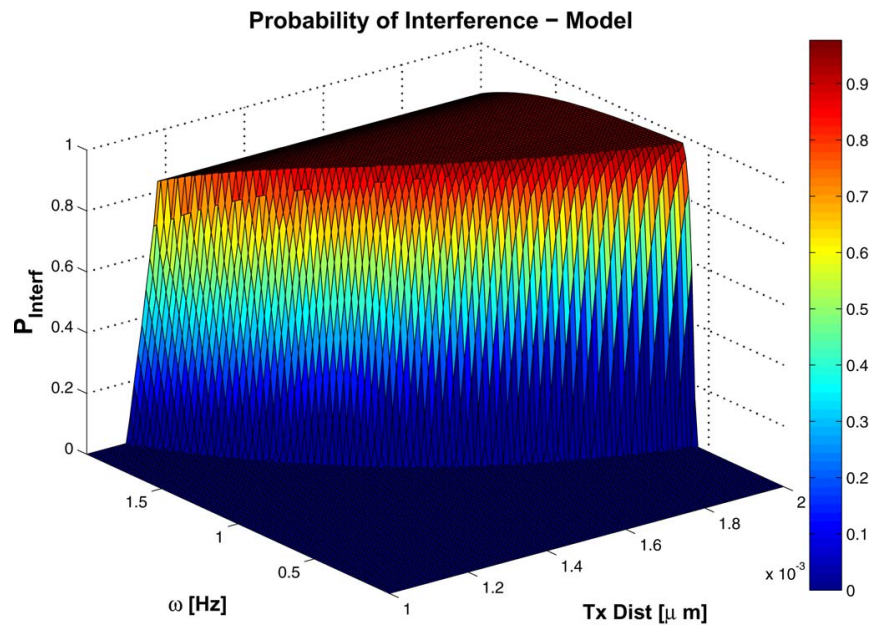

Fig. 5. Probability of interference according to the statistical-physical model.

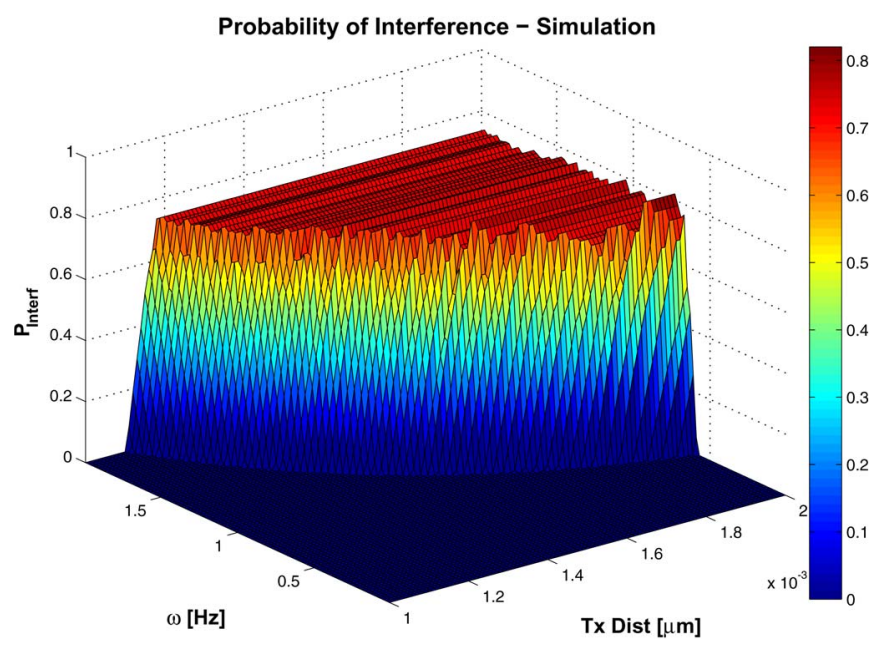

Fig. 6. Probability of interference according to the simulation environment.

of the received PSD $\mathcal{S}_{Y}(\omega)$ computed above with either the statistical-physical model, given by (36), or the simulation environment, given by the numerical results of (37).

In Figs. 5 and 6 we show the probability of interference $P_{\text {Interf }}(\omega)$ according to the statistical-physical model and the simulation environment, respectively, for a range of frequencies $\omega$ from $\omega_{a}=0 \mathrm{~Hz}$ to $\omega_{b}=2 \mathrm{~Hz}$ and for a distance $r_{T x}$ between the single transmitter and the receiver ranging from $1 \mu \mathrm{m}$ to $2 \mu \mathrm{m}$. The values in Fig. 5 are derived from the expression in (42) by using the PDF $P_{\mathcal{S}_{Y}(\omega)}(\omega)$ computed in Section IV-B, while for Fig. 6 we applied the values of the PDF $P_{\mathcal{S}_{Y}\left(q f_{s} / N_{s}\right)}(s)$ computed through the simulation detailed in Section V-A. The constant PSD of the signal $s_{T x}(t)$ is here set to two orders of magnitude higher than the average power of the molecular signals emitted by the interfering transmitters, namely, $\sigma_{t x}^{2}=10^{2} \sigma^{2}$.

In both Figs. 5 and 6 we observe an almost zero probability of interference $P_{\text {Inter } f}(\omega)$ for low values of the frequency $\omega$ and low values for the transmitter distance $r_{T x}$ from the receiver. As the frequency $\omega$ and the distance $r_{T x}$ increase, also the probability of interference $P_{\text {Interf }}(\omega)$ increases from zero to a maximum value. In both Figs. 5 and 6, values of the probability of interference higher than zero occur only for a frequency $\omega$ higher than $0.59 \mathrm{~Hz}$ and a distance $r_{T x}$ higher than $1.1 \mu \mathrm{m}$. In Fig. 5 the maximum value of the probability of interference $P_{\text {Interf }}(\omega)$ is 0.98 and it occurs for the range frequencies $\omega$ between $0.67 \mathrm{~Hz}$ and $0.89 \mathrm{~Hz}$ and for a distance $r_{T x}$ higher than $1.4 \mu \mathrm{m}$. The maximum value of the probability of interference $P_{\text {Interf }}(\omega)$ in Fig. 6 is around 0.82 and it occurs for a frequency $\omega$ around $0.73 \mathrm{~Hz}$ and a distance $r_{T x}$ higher than $1.9 \mu \mathrm{m}$. The overall lower values of the simulation-based probability of interference $P_{\text {Interf }}(\omega)$ in Fig. 6 compared to the values in Fig. 5 from the statistical-physical model are likely due to the limited number of interfering transmitters and iterations of the transmitter distribution realizations considered in the simulation environment, as explained in Section V-A, while the statistical-physical model considers an infinite number of transmitters and it is based on their distribution PDF.

Different behaviors of the probability of interference $P_{\text {Interf }}(\omega)$ for high frequencies $\omega$ and high distances $r_{T x}$ are shown in Figs. 5 and 6 . In the former, the $P_{\text {Interf }}(\omega)$ reaches a plateau, corresponding to the aforementioned maximum value of 0.98 , and then decreases as the frequency value increases from $0.89 \mathrm{~Hz}$ to $2 \mathrm{~Hz}$, where it has a PDF value of 0.83 . In the latter, after a maximum value at 0.82 , and as the frequency increases from $0.73 \mathrm{~Hz}$ to $2 \mathrm{~Hz}$, the $P_{\text {Interf }}(\omega)$ oscillates between 0.74 and 0.72 . Again, this oscillatory behavior is likely due to the limited data used in the simulation environment to compute the PDF $P_{\mathcal{S}_{Y}(\omega)}(s)$, where we considered a limited number of interferers, within a spherical space of radius $\rho=$ $19 \mu \mathrm{m}$, and a limited number of iterations for the realization of their location distribution.

\section{CONCLUSION}

In this paper, we have provided a statistical-physical modeling of the interference in diffusion-based molecular nanonetworks when multiple transmitting nanomachines emit molecules simultaneously. Our method to characterize the interference differentiates from the previous literature since we developed a general model independent from specific modulation and coding techniques. As a result of the property of the received molecular signal of being a stationary Gaussian Process (GP), the statistical-physical modeling has been operated on its Power Spectral Density (PSD), for which it was possible to obtain an analytical expression of the log-characteristic function. This log-characteristic function expression ultimately led to the estimation of the received PSD probability distribution, which provides a complete model of the interference in diffusionbased molecular nanonetworks.

The numerical derivation of the PDF from the logcharacteristic function expression of the received PSD was performed for selected values of the physical parameters of the molecular nanonetwork, such as the diffusion coefficient, the transmitter density, and the average power of molecule emissions. As apparent from the PDF of the received PSD, for low frequencies the power of the received signal tends to a uniform distribution over the range of considered values, while for higher frequencies the power tends with more probability to lower values. 
We evaluated the similarities of the results from the statistical-physical model with the outcomes from simulations, first in terms of received PSD, and then in terms of probability of interference. For the latter comparison, we computed the probability of having at the receiver a contribution from the interference whose PSD exceeds the PSD of a contribution coming from a single transmitter. In both cases, the probability of interference has very low values for frequencies lower than $0.59 \mathrm{~Hz}$ and a distance range lower than $1.1 \mu \mathrm{m}$, while it assumes very high values otherwise. We believe that the statistical-physical model of the interference presented in this paper will be of great help to design the future diffusion-based molecular nanonetworks.

\section{APPENDIX A \\ Chemical Justification of the White Gaussian Model For THE Molecular Signals}

The white Gaussian model for the molecular signals $s_{k}(t)$ expressed in (6) is in agreement with a chemical description of the molecule emission at the molecular transmitters. Without loss of generality, we assume that the total molecule concentration $c_{\text {base }}+c\left(\bar{x}_{k}, t\right)$ at each transmitter $k$ is a function of $M$ different chemical reactions involving $N$ different chemical species (molecule types) within the transmitter definite volume $V_{T}$. According to the chemical Langevin equation approximation [20], the time derivative $d X_{n}(t) / d t$ from (1) in the number $X_{n}$ of species- $n$ molecules, and function of the time $t$, is given by the following expression:

$$
\frac{d X_{n}(t)}{d t}=\sum_{m=1}^{M} \nu_{m n} a_{m}(\mathbf{X}(t))+\sum_{m=1}^{M} \nu_{m n} \sqrt{a_{m}(\mathbf{X}(t))} \Gamma_{m}(t)
$$

where $\mathbf{X}(t)=\left[X_{1}(t), X_{2}(t), \ldots, X_{N}(t)\right]^{\prime}$ is a vector that contains the number of molecules of each reacting species, $\nu_{m n}$ corresponds to the change in the number of molecules of the chemical species $n$ produced by the chemical reaction $m$, $a_{m}(\mathbf{X}(t))$, which is called propensity function, is the probability that the chemical reaction $m$ will occur within the transmitter volume as function of the vector $\mathbf{X}(t)$, and $\Gamma_{m}(t)$ are i.i.d. white Gaussian signals. Under the assumption to have the chemical reactions at equilibrium within every transmitter volume, which is expressed as $\sum_{m=1}^{M} \nu_{m n} a_{m}(\mathbf{X}(t))=0$, and given (43), the molecular signal $s_{k}(t)$ in (1) is equal to a sum of i.i.d. white Gaussian signals as follows:

$$
s_{k}(t)=\frac{1}{V_{T}} \sum_{m=1}^{M} \nu_{m n} \sqrt{a_{m}(\mathbf{X}(t))} \Gamma_{m}(t) .
$$

As a consequence of the property of a linear combination of i.i.d. Gaussian random variables [28], $s_{k}(t)$, as expressed in (6), is a white Gaussian signal with zero mean and variance $\sigma^{2}$ equal to

$$
\sigma^{2}=\frac{1}{V_{T}^{2}} \sum_{m=1}^{M} \nu_{m n}^{2} a_{m}(\mathbf{X}(t))
$$

where $\mathbf{X}(t)$ is the vector that contains the number of molecules of each reacting species, $\nu_{m n}$ corresponds to the change in the number of molecules of the chemical species $n$ produced by the chemical reaction $m$, and the propensity function $a_{m}(\mathbf{X}(t))$ is the probability that the chemical reaction $m$ will occur within the transmitter volume as function of the vector $\mathbf{X}(t)$.

\section{ACKNOWLEDGMENT}

The authors would like to thank A. Ozan Bicen for his constructive and detailed feedback, which helped to improve the quality of the paper.

\section{REFERENCES}

[1] I. F. Akyildiz, F. Brunetti, and C. Blazquez, "Nanonetworks: A new communication paradigm at molecular level," Comput. Netw., vol. 52, no. 12, pp. 2260-2279, Aug. 2008.

[2] I. F. Akyildiz, J. M. Jornet, and M. Pierobon, "Nanonetworks: A new frontier in communications," Commun. ACMs, vol. 54, no. 11, pp. 84-89, Nov. 2011

[3] L. Parcerisa and I. F. Akyildiz, "Molecular communication options for long range nanonetworks," Comput. Netw., vol. 53, no. 16, pp. 27532766, Aug. 2009.

[4] M. Moore, A. Enomoto, T. Nakano, R. Egashira, T. Suda, A. Kayasuga, H. Kojima, H. Sakakibara, and K. Oiwa, "A design of a molecular communication system for nanomachines using molecular motors," in Proc. 4th Annu. IEEE Int. Conf. Pervasive Comput. Communications Workshops, Mar. 2006, pp. 6-12.

[5] M. Gregori and I. F. Akyildiz, "A new nanonetwork architecture using flagellated bacteria and catalytic nanomotors," IEEE J. Sel. Areas Commun., vol. 28, no. 4, pp. 602-611, May 2010.

[6] S. Kadloor and R. Adve, "A framework to study the molecular communication system," in Proc. 18th Int. Conf. Comput. Commun. Netw., Aug. 2009, pp. 1-6.

[7] M. J. Moore, T. Suda, and K. Oiwa, "Molecular communication: Modeling noise effects on information rate," IEEE Trans. Nanobiosci., vol. 8, no. 2, pp. 169-180, Jun. 2009.

[8] D. J. Spencer, S. K. Hampton, P. Park, J. P. Zurkus, and P. J. Thomas, "The diffusion-limited biochemical signal-relay channel," Adv. Neural Inf. Process. Syst., vol. 16, pp. 1263-1270, 2004.

[9] B. Atakan and O. B. Akan, "An information theoretical approach for molecular communication," in Proc. 2nd Conf. Bio-Inspired Models Netw., Inf. Comput. Syst., Dec. 2007, pp. 33-40.

[10] B. Atakan and O. B. Akan, "On molecular multiple-access, broadcast, relay channels in nanonetworks," in Proc. 3rd Int. Conf. Bio-Inspired Models Netw., Inf. Comput. Syst., Nov. 2008, pp. 16:1-16:8.

[11] I. F. Akyildiz, F. Fekri, R. Sivakumar, C. R. Forest, and B. K. Hammer, "MoNaCo: Fundamentals of molecular nano-communication networks," IEEE Wireless Commun. Mag., vol. 19, no. 5, pp. 12-18, Oct. 2012.

[12] M. Pierobon and I. F. Akyildiz, "A physical end-to-end model for molecular communication in nanonetworks," IEEE J. Sel. Areas Commun., vol. 28, no. 4, pp. 602-611, May 2010.

[13] M. Pierobon and I. F. Akyildiz, "Diffusion-based noise analysis for molecular communication in nanonetworks," IEEE Trans. Signal Process., vol. 59, no. 6, pp. 2532-2547, Jun. 2011.

[14] M. Pierobon and I. F. Akyildiz, "Noise analysis in ligand-binding reception for molecular communication in nanonetworks," IEEE Trans. Signal Process., vol. 59, no. 9, pp. 4168-4182, Sep. 2011.

[15] M. Pierobon and I. F. Akyildiz, "Capacity of a diffusion-based molecular communication system with channel memory and molecular noise," IEEE Trans. Inf. Theory, vol. 59, no. 2, pp. 942-954, Feb. 2013.

[16] M. S. Kuran, H. B. Yilmaz, T. Tugcu, and I. F. Akyildiz, "Interference effects on modulation techniques in diffusion based nanonetworks," Nano Commun. Netw., vol. 3, no. 1, pp. 65-73, Mar. 2012.

[17] M. Mahfuz, D. Makrakis, and H. Mouftah, "Characterization of intersymbol interference in concentration-encoded unicast molecular communication," in Proc. CCECE, May 2011, pp. 000164-000168.

[18] B. Atakan, S. Galmes, and O. Akan, "Nanoscale communication with molecular arrays in nanonetworks," IEEE Trans. NanoBiosci., vol. 11, no. 2, pp. 149-160, Jun. 2012. 
[19] M. Pierobon and I. F. Akyildiz, "Intersymbol and co-channel interference in diffusion-based molecular communication," in Proc. 2nd IEEE Int. Workshop MoNaCom, ICC, Jun. 2012, pp. 6126-6131.

[20] D. T. Gillespie, "The chemical Langevin equation," J. Chem. Phys., vol. 113, no. 1, pp. 297-306, Jul. 2000.

[21] C. J. Myers, Engineering Genetic Circuits, Mathematical and Computational Biology Series. Boston, MA, USA: Chapman \& Hall, 2009.

[22] D. L. Nelson and M. M. Cox, Lehninger Principles of Biochemistry. San Francisco, CA, USA: Freeman, 2005, ch. 12.2, pp. 425-429.

[23] J. Philibert, "One and a half century of diffusion: Fick, Einstein, before and beyond," Diffusion Fundam., vol. 4, pp. 6.1-6.19, 2006.

[24] E. L. Cussler, Diffusion. Mass Transfer in Fluid Systems, 2nd ed. Cambridge, U.K.: Cambridge Univer. Press, 1997.

[25] B. S. Donahue and R. F. Abercrombie, "Free diffusion coefficient of ionic calcium in cytoplasm," Cell Calcium, vol. 8, no. 6, pp. 437-448, Dec. 1987.

[26] S. Jeanson, J. Chadœuf, M. N. Madec, S. Aly, J. Floury, T. F. Brocklehurst, and S. Lortal, "Spatial distribution of bacterial colonies in a model cheese," Appl. Environ. Microbiol., vol. 77, no. 4, pp. 1493-1500, Feb. 2011.

[27] D. Heath, An Introduction To Experimental Design And Statistics For Biology. Boca Raton, FL, USA: CRC Press, 2002.

[28] A. Papoulis and S. U. Pillai, Probability, Random Variables and Stochastic Processes, 4th ed. New York, NY, USA: McGraw-Hill, 2002.

[29] B. Davies, Integral Transforms and Their Applications. New York, NY, USA: Springer-Verlag, 2002.

[30] A. Mandelis, Diffusion-Wave Fields: Mathematical Methods and Green Functions. New York, NY, USA: Springer-Verlag, 2001.

[31] J. Ilow and D. Hatzinakos, "Analytic alpha-stable noise modeling in a Poisson field of interferers or scatterers," IEEE Trans. Signal Process., vol. 46, no. 6, pp. 1601-1611, Jun. 1998.

[32] K. Gulati, B. L. Evans, J. G. Andrews, and K. R. Tinsley, "Statistics of cochannel interference in a field of Poisson and Poisson-Poisson clustered interferers," IEEE Trans. Signal Process., vol. 58, no. 12, pp. 6207-6222, Dec. 2010.

[33] M. Abramowitz and I. A. Stegun, Handbook of Mathematical Functions With Formulas, Graphs, Mathematical Tables. New York, NY, USA: Dover Publications, 1972

[34] B. Novak and J. Tyson, "Design principles of biochemical oscillators," Nature Rev. Mol. Cell Biol., vol. 9, no. 12, pp. 981-991, Dec. 2008.

[35] P. Maquet, C. Degueldre, G. Delfiore, J. Aerts, J. M. Peters, A. Luxen, and G. Franck, "Functional neuroanatomy of human slow wave sleep," J. Neurosci., vol. 17, no. 8, pp. 2807-2812, Aug. 1997.

[36] J. S. Simonoff, Smoothing Methods in Statistics. New York, NY, USA: Springer-Verlag, 1998, S. S. in Statistics, Ed.

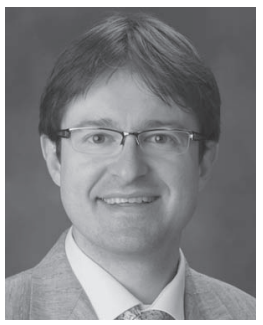

Massimiliano Pierobon (S'09-M'09) received the B.S.+M.S. degree in telecommunication engineering from the Politecnico di Milano, Milan, Italy, in 2005 and the Ph.D. degree in electrical and computer engineering from the Georgia Institute of Technology, Atlanta, GA, USA, in August 2013.

$\mathrm{He}$ is currently an Assistant Professor with the Department of Computer Science \& Engineering at the University of Nebraska-Lincoln, Lincoln, NE, USA. During 2006, he worked as a researcher in the R\&D department of Siemens Carrier Networks, Milan, where he coauthored two filed patents on jitter buffer management. From January 2007 to July 2009 he was a graduate Research Assistant at the Politecnico di Milano in the fields of signal processing and pattern recognition. In November 2008 he joined the BWN lab, first as a visiting researcher and, from August 2009, as a Ph.D. student. He received the BWN Lab Researcher of the Year Award at the Georgia Institute of Technology for his outstanding research achievements in 2011. He was also named IEEE COMMUNICATIONS LETTERS 2013 Exemplary Reviewer in appreciation for his service as referee. $\mathrm{He}$ is a member of ACM and ACS. His current research interests are in molecular communication theory for nanonetworks, communication engineering applied to intelligent drug delivery systems and biological circuit network engineering for microbial communication networks.

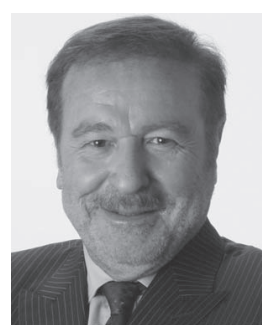

Ian F. Akyildiz (F'96) received the B.S., M.S., and $\mathrm{Ph} . \mathrm{D}$. degrees in computer engineering from the University of Erlangen-Nürnberg, Erlangen, Germany, in 1978, 1981, and 1984, respectively.

Currently, he is the Ken Byers Chair Professor in Telecommunications with the School of Electrical and Computer Engineering, Georgia Institute of Technology, Atlanta, GA, USA, the Director of the Broadband Wireless Networking (BWN) Laboratory, and the Chair of the Telecommunication Group at Georgia Tech. Since 2013, he is a FiDiPro Professor (Finland Distinguished Professor Program (FiDiPro) supported by the Academy of Finland) in the Department of Electronics and Communications Engineering, at Tampere University of Technology, Tampere, Finland, and the founding director of NCC (Nano Communications Center). Since 2008, he is also an honorary professor with the School of Electrical Engineering at Universitat Politécnica de Catalunya (UPC) in Barcelona, Catalunya, Spain and the founding director of N3Cat (NaNoNetworking Center in Catalunya). Since 2011, he is a Consulting Chair Professor at the Department of Information Technology, King Abdulaziz University (KAU) in Jeddah, Saudi Arabia. He is the Editor-in-Chief of Computer Networks (Elsevier) Journal, and the founding Editor-in-Chief of the Ad Hoc Networks (Elsevier) Journal, the Physical Communication (Elsevier) Journal and the Nano Communication Networks (Elsevier) Journal. He is an IEEE Fellow (1996) and an ACM Fellow (1997). He received numerous awards from IEEE and ACM. His current research interests are in nanonetworks, Terahertz Band communication networks, Long Term Evolution Advanced (LTE-A) networks, cognitive radio networks and wireless sensor networks. 